\title{
Validation of EGSYS Score in Prediction of Cardiogenic Syncope
}

\author{
Hamid Kariman, ${ }^{1}$ Sepideh Harati, ${ }^{1}$ Saeed Safari, ${ }^{2}$ Alireza Baratloo, ${ }^{2}$ and Mehdi Pishgahi ${ }^{3}$ \\ ${ }^{1}$ Emergency Department, Imam Hossein Hospital, Shahid Beheshti University of Medical Sciences, Tehran 19899 34148, Iran \\ ${ }^{2}$ Emergency Department, Shohadaye Tajrish Hospital, Shahid Beheshti University of Medical Sciences, Tehran 19899 34148, Iran \\ ${ }^{3}$ Cardiology Department, Shohadaye Tajrish Hospital, Shahid Beheshti University of Medical Sciences, Tehran 19899 34148, Iran
}

Correspondence should be addressed to Saeed Safari; safari266@gmail.com

Received 14 September 2015; Revised 18 October 2015; Accepted 1 November 2015

Academic Editor: Robert W. Derlet

Copyright (C) 2015 Hamid Kariman et al. This is an open access article distributed under the Creative Commons Attribution License, which permits unrestricted use, distribution, and reproduction in any medium, provided the original work is properly cited.

\begin{abstract}
Introduction. Evaluation of Guidelines in Syncope Study (EGSYS) is designed to differentiate between cardiac and noncardiac causes of syncope. The present study aimed to evaluate the accuracy of this predictive model. Methods. In this prospective cross-sectional study, screening performance characteristics of EGSYS-U (univariate) and EGSYS-M (multivariate) in prediction of cardiac syncope were calculated for syncope patients who were referred to the emergency department (ED). Results. 198 patients with mean age of $59.26 \pm 19.5$ years were evaluated (62.3\% male). 115 (58.4\%) patients were diagnosed with cardiac syncope. Area under the ROC curve was 0.818 (95\% CI: 0.75-0.87) for EGSYS-U and 0.805 (CI 95\%: 0.74-0.86) for EGSYS-M ( $p=0.53$ ). Best cut-off point for both models was $\geq 3$. Sensitivity and specificity were $86.08 \%$ (95\% CI: 78.09-91.59) and $68.29 \%$ (95\% CI: 56.97-77.86) for EGSYS-U and $91.30 \%$ (95\% CI: 84.20-95.52) and 57.32\% (95\% CI: 45.92-68.02) for EGSYS-M, respectively. Conclusion. The results of this study demonstrated the acceptable accuracy of EGSYS score in predicting cardiogenic causes of syncope at the $\geq 3$ cut-off point. It seems that using this model in daily practice can help physicians select at risk patients and properly triage them.
\end{abstract}

\section{Introduction}

Syncope is a transient loss of consciousness and postural tone, which is responsible for about $5 \%$ of emergency department (ED) referrals [1]. This symptom has a wide range of causes from nonserious to potentially fatal [2-8]. Accurate cause prediction and choosing the proper approach to these patients have been historical challenges for physicians [9]. Causes of syncope can be broadly divided into cardiac and noncardiac. Usually, patients with cardiogenic syncope have a higher mortality rate regardless of their age [2]. Clinical and electrocardiography (ECG) findings can be helpful in the classification of syncope with cardiac origin $[1,10]$. In some developed countries, syncope units are responsible for the management of these patients, which have efficiently reduced unnecessary hospitalization $[1,4,5]$. Yet, in most countries, these units cannot be established and simpler strategies are used for triage of these patients. One of these strategies is using clinical risk stratification rules such as San Francisco syncope rule, short-term prognosis of syncope (STePS), and Evaluation of Guidelines in Syncope Study (EGSYS) [5, 9, 1118]. Most of these models predict the patients' outcome, while
EGSYS has been designed to differentiate between cardiac and noncardiac causes of syncope. In this model, patients are given a score based on the presence or absence of risk factors such as abnormal ECG findings, palpitation, precipitating or predisposing factors, and autonomic prodromes. Higher scores are in favor of cardiac causes. Previous studies have introduced EGSYS as an efficient and sensitive tool in the prediction of cardiogenic syncope [3, 12]. In a cohort study by Del Rosso et al. sensitivity and specificity of this model were reported to be $92 \%$ and $69 \%$, respectively [12]. Based on the above-mentioned points, the present study was designed to validate EGSYS scores in the differentiation of cardiac and noncardiac causes of syncope.

\section{Methods}

This prospective cross-sectional validation study was carried out on syncope patients who were referred to ED of three University Hospitals, Tehran, Iran, from March 2012 to March 2013. This study was aimed to evaluate the accuracy of EGSYS scores in the prediction of cardiogenic syncope. Protocol 
of the present study was approved by the ethics committee of Shahid Beheshti University of Medical Sciences and the researchers adhered to the principles of Helsinki Declaration. Informed written consent was obtained from all of the studied patients. All patients $>18$ years old, who were referred within 24 hours of symptom initiation, were included. The subjects were enrolled using nonprobability consecutive sampling by Sepideh Harati during her routine 12-hour residency shifts (day or night) under direct supervision of attending physician on duty. Patients, who did not give their consent or the origin of their syncope was unknown, were excluded. Initially, enrolled patients were physically examined by the senior emergency medicine resident (Sepideh Harati) and their demographic data (age, sex) as well as EGSYS risk factors (Table 2) were recorded using a predesigned checklist [1]. Then, the patients were followed until their final cause of syncope (cardiac or noncardiac) was determined. For patients whose cause of syncope was clear during their hospitalization, data were recorded from patients' files. While cases, that were discharged before confirmation of diagnosis and scheduled for additional outpatient tests such as electrophysiological study, which was not available in the studied centers, were followed using telephone call with patients or their in charge family physicians. Therefore, follow-up period varied from one week to one month in some cases. Next, univariate and multivariate EGSYS scores were calculated for all the patients. The patients' ECGs were interpreted by an independent cardiologist who was blind to the clinical status and diagnosis of the patient. Final decision on the probable cardiac cause of syncope was made based on the results of echocardiography, stress testing, prolonged Holter ECG monitoring, and electrophysiological study. In cases of normal cardiac evaluations, probable neurologic causes were ruled out using tilt testing, brain imaging, and carotid massage [7]. The origin was reported as unknown if the above-mentioned evaluations were normal.

2.1. Calculation of EGSYS Score. At first, the presence of risk factors listed in Table 2 was evaluated for each patient. Then, the sum of the scores was calculated for each patient. Those with a total score $\geq 3$ were considered at risk for presence of cardiac cause.

2.2. Definitions. Since the present research aimed to validate the study by Del Rosso et al. [12], all terms and definitions were based on the original derivation study.

(i) ECG abnormality was considered as the presence of one or more of the following abnormalities: bradycardia $(<40$ beat/minute), ST changes ( $>1 \mathrm{~mm}$ elevation or depression), QT prolongation (440 ms), ventricular tachycardia, atrioventricular block (second or third degree), sick sinus syndrome, ventricular and rapid paroxysmal supraventricular arrhythmias, sinus pauses, and pace malfunction.

(ii) Predisposing or precipitating factors were considered as the presence of one or more of the following abnormalities: warm place, crowded place, prolonged
TABLE 1: Baseline characteristics of the studied patients.

\begin{tabular}{lc}
\hline Variable & Values \\
\hline Systolic blood pressure (mmHg) & \\
Maximum & $123.87 \pm 26.72$ \\
Minimum & $74.87 \pm 12.06$ \\
Pulse rate (per minute) & $75.87 \pm 18.75$ \\
$\mathrm{O}_{2}$ saturation $(\%)$ & $95.57 \pm 4.16$ \\
Hemoglobin (mg/dL) & $12.63 \pm 1.5$ \\
Tilt positive & $11(5.6)$ \\
ECG abnormality & $71(36.2)$ \\
T-changes (elevation, depression) & $44(22.6)$ \\
ST changes ( $>1$ mm) & $14(7.2)$ \\
QT $>440$ milliseconds & $21(14.3)$ \\
Atrioventricular block & $3(1.6)$ \\
QRS $>120$ milliseconds & $32(25.4)$ \\
History of syncope & \\
No & $142(73.6)$ \\
Yes & $46(23.8)$ \\
Occurrence of previous syncope & \\
$\leq 1$ month & $42(91.3)$ \\
$<1$ month & $4(8.7)$ \\
$<1$ week & $32(69.6)$ \\
Position & \\
Supine & $19(9.9)$ \\
Upright & $116(60.4)$ \\
Sitting & $33(17.2)$ \\
Activity during syncope & \\
Effort & $68(35.8)$ \\
Resting & $54(28.4)$ \\
\hline
\end{tabular}

Values were presented as mean \pm standard deviation or number and percentage.

standing, overtiring, postprandial period, neck turning, syncope while sitting, supine, or upright, syncope during effort, and syncope after effort.

(iii) Prodromal symptoms and signs were considered as the presence of one or more of the following abnormalities: blurred vision, lightheadedness, diaphoresis, palpitations, nausea, vomiting, abdominal discomfort, weakness, feeling cold, feeling warm, tremors, yawn, pallor, redness, or cyanosis.

2.3. Statistical Analysis. Considering 90\% sensitivity, 5\% desired precision, and 95\% confidence interval (CI), the minimum sample size was calculated to be 138 cases. Screening performance characteristics of EGSYS score (sensitivity, specificity, positive and negative predictive values, and positive and negative likelihood ratios) in prediction of cardiogenic syncope were calculated using SPSS version 21.0. Receiver operating characteristic (ROC) and area under the curve with 95\% CI were used to find the best cut-off point and accuracy of the model. $p<0.05$ was considered significant.

\section{Results}

206 patients were evaluated; however, 8 cases with mean age of $50.0 \pm 22.74(75.0 \%$ male $)$ were excluded due to unknown origin of syncope. No cases of miss or death happened during 
TABLE 2: Frequency of EGSYS risk factors in the studied patients.

\begin{tabular}{|c|c|c|c|c|}
\hline Risk factors & Number (\%) & $\mathrm{OR}^{3}\left(95 \% \mathrm{CI}^{4}\right)$ & $p$ value & Score $^{\#}$ \\
\hline \multicolumn{5}{|l|}{ EGSYS-U $\mathrm{U}^{1}$} \\
\hline Abnormal ECG*/cardiopathy & $134(67.3)$ & $11.27(5.42-23.01)$ & $<0.001$ & 3 \\
\hline Palpitations/dyspnea & $59(30.1)$ & $7.48(3.3-16.96)$ & $<0.001$ & 3 \\
\hline Syncope in supine position/effort syncope & $68(34.2)$ & $1.37(0.74-2.40)$ & 0.315 & 2 \\
\hline Age $>64$ years & $87(45.1)$ & $4.54(2.24-8.53)$ & $<0.001$ & 1 \\
\hline No precipitating and predisposing factors & $128(65.6)$ & $0.84(0.46-1.53)$ & 0.576 & 1 \\
\hline No prodromes ${ }^{* *}$ & $46(23.6)$ & $3.84(1.73-8.52)$ & 0.001 & 1 \\
\hline Blurred vision & $40(20.7)$ & $5.12(2.37-11.06)$ & $<0.001$ & -1 \\
\hline Neurovegetative signs during recovery phase & $68(35.2)$ & $2.19(1.20-4.01)$ & 0.010 & -1 \\
\hline Precipitating and predisposing factors ${ }^{* * *}$ & $67(34.4)$ & $0.84(0.46-1.53)$ & 0.575 & -2 \\
\hline Autonomic prodromes & $67(34.2)$ & $0.13(0.02-1.05)$ & 0.055 & -2 \\
\hline \multicolumn{5}{|l|}{ EGSYS-M ${ }^{2}$} \\
\hline Abnormal ECG/cardiopathy & $134(67.3)$ & $11.27(5.42-23.01)$ & $<0.001$ & 3 \\
\hline Palpitations/dyspnea & $59(30.1)$ & $7.48(3.3-16.96)$ & $<0.001$ & 4 \\
\hline Effort syncope & $51(26.4)$ & $0.94(0.49-1.79)$ & 0.844 & 3 \\
\hline Syncope in supine position & $9(9.8)$ & $4.28(1.20-15.25)$ & 0.016 & 2 \\
\hline Autonomic prodromes & $67(34.2)$ & $0.13(0.02-1.05)$ & 0.025 & -1 \\
\hline Precipitating and predisposing factors & $67(34.4)$ & $0.84(0.46-1.53)$ & 0.575 & -1 \\
\hline
\end{tabular}

* Presence of one or more ECG abnormality listed in Definitions.

** Defined in Methods.

*** Defined in Methods.

\# Total score $\geq 3$ indicates patients at risk for presence of cardiac cause.

${ }^{1}$ EGSYS-U: Evaluation of Guidelines in Syncope Study-Univariate.

${ }^{2}$ EGSYS-M: Evaluation of Guidelines in Syncope Study-Multivariate.

${ }^{3} \mathrm{OR}$ : odds ratio.

${ }^{4} \mathrm{CI}$ : confidence interval.

TABLE 3: The comparison of EGSYS risk factors between cardiac and noncardiac causes of syncope.

\begin{tabular}{|c|c|c|c|}
\hline \multirow{2}{*}{ EGSYS* } & \multicolumn{2}{|c|}{ Cause of syncope } & \multirow{2}{*}{$p$ value } \\
\hline & Noncardiac $N(\%)$ & Cardiac N (\%) & \\
\hline Abnormal ECG/cardiopathy & $32(24.1)$ & $101(75.9)$ & 0.010 \\
\hline Palpitations/dyspnea & $8(13.6)$ & $51(86.4)$ & $<0.001$ \\
\hline Syncope in supine position/effort syncope & $25(36.8)$ & $43(63.2)$ & 0.315 \\
\hline Age $>64$ years & $20(23)$ & $67(77)$ & $<0.001$ \\
\hline No precipitating and predisposing factors & $55(43)$ & $73(57)$ & 0.575 \\
\hline No prodromes & $9(19.6)$ & $37(80.4)$ & $\leq 0.001$ \\
\hline Blurred vision & $29(72.5)$ & $11(27.5)$ & $<0.001$ \\
\hline Neurovegetative signs during recovery phase & $37(54.4)$ & $31(45.6)$ & 0.010 \\
\hline Precipitating and predisposing factors & $26(38.8)$ & $41(61.2)$ & 0.575 \\
\hline Neurovegetative prodromes & $1(9.1)$ & $10(90.1)$ & 0.025 \\
\hline
\end{tabular}

${ }^{*}$ Evaluation of Guidelines in Syncope Study-Univariate.

the patients' follow-up period. The EGSYS-M score of all excluded patients were $\leq 2$. Finally, 198 patients with mean age of $59.26 \pm 19.5$ years (range: $13-98$ ) were enrolled in the study $(62.3 \%$ male). Table 1 shows baseline characteristics of the participants. Frequency of EGSYS risk factors is shown in Table 2.115 (58.4\%) patients were diagnosed with cardiac and $83(41.6 \%)$ with noncardiac origin. The comparison of EGSYS risk factors between cardiac and noncardiac syncope can be found in Table 3. Mean EGSYS-U and EGSYS-M scores were $3.57 \pm 3.05$ (minimum -4 and maximum 10 ) and $3.73 \pm 2.68$ (minimum -2 and maximum 10), respectively. Mean EGSYS$\mathrm{M}$ and EGSYS-U scores for cardiac and noncardiac causes were $4.9 \pm 2.1$ and $2.11 \pm 2.4(p<0.001)$ and $5 \pm 2.5$ and $1.6 \pm$ $2.6(p<0.001)$, respectively. Figure 1 shows the comparative ROC curve of the 2 mentioned models. Area under the ROC curve was 0.818 (95\% CI: 0.75-0.87) for EGSYS-U and 0.805 (CI 95\%: 0.74-0.86) for EGSYS-M ( $p=0.53$ ). Best cut-off point for both models was estimated to be $\geq 3$. Table 4 summarizes screening performance characteristics of the 2 mentioned models. At $\geq 3$ cut-off point, sensitivity and 
TABLE 4: Screening performance characteristics of EGSYS- $\mathrm{U}^{1}$ and EGSYS-M ${ }^{2}$ in prediction of cardiac causes of syncope (cut-off point $\geq 3$ ).

\begin{tabular}{lcc}
\hline $\begin{array}{l}\text { Screening } \\
\text { performance } \\
\text { characteristics }\end{array}$ & EGSYS-U $\left(95 \% \mathrm{CI}^{3}\right)$ & EGSYS-M $(95 \% \mathrm{CI})$ \\
\hline $\begin{array}{l}\text { Sensitivity } \\
\text { Specificity }\end{array}$ & $86.08(78.09-91.59)$ & $91.30(84.20-95.52)$ \\
$\begin{array}{l}\text { Positive predictive } \\
\text { value }\end{array}$ & $79.20(70.83-85.73)$ & $57.00(66.84-81.75)$ \\
$\begin{array}{l}\text { Negative predictive } \\
\text { value }\end{array}$ & $77.78(66.15-86.39)$ & $82.46(69.64-90.82)$ \\
$\begin{array}{l}\text { Positive likelihood } \\
\text { ratio }\end{array}$ & $3.81(2.67-5.42)$ & $3.00(2.22-4.06)$ \\
$\begin{array}{l}\text { Negative likelihood } \\
\text { ratio }\end{array}$ & $0.29(0.18-0.44)$ & $0.21(0.12-0.38)$ \\
\hline
\end{tabular}

${ }^{1}$ EGSYS-U: Evaluation of Guidelines in Syncope Study-Univariate.

${ }^{2}$ EGSYS-M: Evaluation of Guidelines in Syncope Study-Multivariate.

${ }^{3} \mathrm{CI}$ : confidence interval.

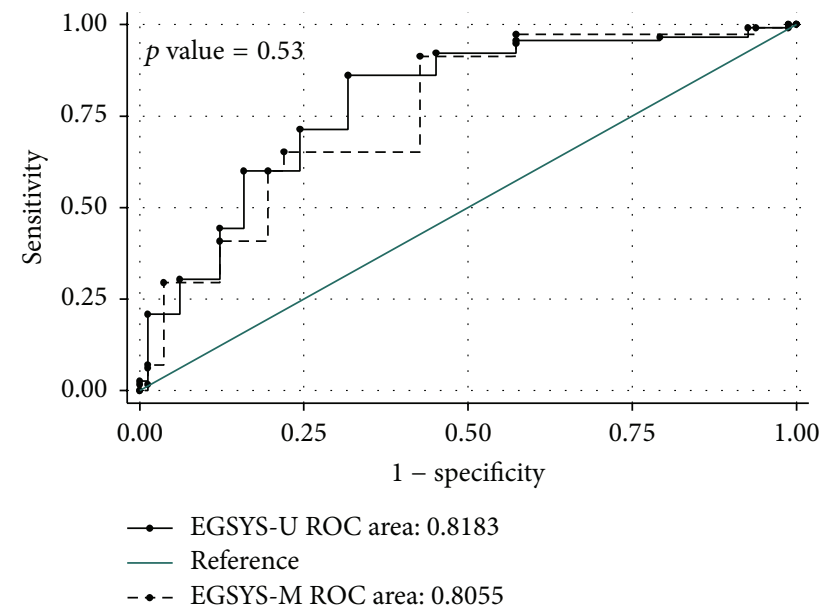

FIGURE 1: Comparative receiver operative characteristic (ROC) curve of univariate EGSYS (EGSYS-U) and multivariate EGSYS (EGSYS-M).

specificity were $86.08 \%$ (95\% CI: 78.09-91.59) and $68.29 \%$ (95\% CI: 56.97-77.86) for EGSYS-U and 91.30\% (95\% CI: 84.20-95.52) and 57.32\% (95\% CI: 45.92-68.02) for EGSYS$\mathrm{M}$, respectively.

\section{Discussion}

Based on the results of this study, EGSYS score at $\geq 3$ cut-off point shows acceptable sensitivity for screening the patients with cardiogenic syncope. No significant difference was seen between the univariate and multivariate models in this regard. Mean EGSYS score of the patients with cardiac causes was significantly higher (about twice as much). The value of clinical findings and characteristics of syncope in prediction of patient outcomes have been evaluated in different studied $[12,18,19]$. In addition, various clinical decision rules have been designed and validated for this purpose $[5,9,11-$ 18]. Among them, EGSYS claims to be able to differentiate between cardiac causes and noncardiac causes of syncope. A study on 153 patients in 2008 showed that EGSYS is an efficient tool to distinguish cardiogenic syncope, reducing unnecessary hospitalization and improving ED management [18]. A study by Del Rosso et al. in 2008 also demonstrated that EGSYS has high sensitivity in predicting cardiac causes of syncope [12]. One-month and 2-year outcome assessment of 465 syncope patients in 2010 (EGSYS-2 study) demonstrated an increased mortality rate in patients with higher EGSYS scores [19]. The results of one cohort study estimated the sensitivity of EGSYS in predicting mortality and bad outcome to be $80 \%$ and $56 \%$, respectively [20]. In the present study, best cut-off point for differentiating cardiac causes of syncope for both univariate and multivariate models was $\geq 3$, which is in line with the results of the previous studies $[12,18]$. Area under the ROC curve in the present study for both models was above $80 \%$, which represents a good level of accuracy. Among the EGSYS risk factors, highest odds ratios belonged to ECG abnormality (11.27), palpitation (7.48), and blurred vision (5.12) which were lower than these values in the Del Rosso et al. study, except for ECG abnormality. Regarding the high percentage of cardiogenic causes in comparison to previous studies $[12,21]$, two points should be mentioned: (1) the sampling is not census and (2) the end point for data gathering was reaching at least 138 cases of cardiac syncope; therefore, this finding is not relevant to prevalence of cardiogenic syncope.

Limitations. Sample size for the present study was estimated to be 138 cases of cardiogenic syncope, but, in the end, the study was done with 115 cases. Since the power of this study was $99.99 \%$ based on comparing the mean EGSYS score in cardiac and noncardiac groups, the aforementioned deficiency has no effect on the results of study. On the other hand, it should be considered that some syncope patients are never referred to ED due to rapid improvement of clinical status, most of which have probably had benign noncardiac causes. This can shift the composition of the participants in favor of cardiogenic causes and affect the results. Therefore, we need comprehensive multicentric studies to be able to generalize the findings. In addition, evaluating the short- and long-term outcome of the patients can aid in making more adequate and accurate decisions.

\section{Conclusion}

The results of this study demonstrated the acceptable accuracy of EGSYS score in predicting cardiogenic causes of syncope at the $\geq 3$ cut-off point. There was no significant difference between the univariate and multivariate models in this regard. It seems that using this prediction model in daily practice can help physicians in the selection of at risk patients and proper triage of them for further evaluations. 


\section{Key Elements}

One can find the following.

(1) EGSYS score can predict cardiogenic causes of syncope with acceptable accuracy.

(2) The best cut point of model for this purpose is score $\geq 3$.

(3) There is no significant difference between the univariate and multivariate models.

(4) It could be helpful in triage of syncope patients.

\section{Disclosure}

This paper has been extracted from Dr. Sepideh Haratis specialist thesis.

\section{Conflict of Interests}

The authors declare that there is no conflict of interests.

\section{Authors' Contribution}

All authors met four recommended criteria of authorship by International Committee of Medical Journal Editors (ICMJE).

\section{Acknowledgments}

The authors wish to thank all the medical staff and personnel of Emergency Departments at Imam Hossein, Loghman Hakim, and Shohadaye Tajrish Hospitals, who contributed to different parts of the study.

\section{References}

[1] M. Brignole, P. Alboni, D. G. Benditt et al., "Guidelines on management (diagnosis and treatment) of syncope-update 2004. The task force on syncope, European Society of Cardiology," European Heart Journal, vol. 25, no. 22, pp. 2054-2072, 2004.

[2] E. S. Soteriades, J. C. Evans, M. G. Larson et al., "Incidence and prognosis of syncope," The New England Journal of Medicine, vol. 347, no. 12, pp. 878-885, 2002.

[3] M. Brignole, M. Disertori, C. Menozzi et al., "Management of syncope referred urgently to general hospitals with and without syncope units," Europace, vol. 5, no. 3, pp. 293-298, 2003.

[4] R. A. Kenny, D. O'Shea, and H. F. Walker, "Impact of a dedicated syncope and falls facility for older adults on emergency beds," Age and Ageing, vol. 31, no. 4, pp. 272-275, 2002.

[5] W. K. Shen, W. W. Decker, P. A. Smars et al., "Syncope Evaluation in the Emergency Department Study (SEEDS): a multidisciplinary approach to syncope management," Circulation, vol. 110, no. 24, pp. 3636-3645, 2004.

[6] F. Colivicchi, F. Ammirati, D. Melina, V. Guido, G. Imperoli, and M. Santini, "Development and prospective validation of a risk stratification system for patients with syncope in the emergency department: The OESIL risk score," European Heart Journal, vol. 24, no. 9, pp. 811-819, 2003.
[7] A. Moya, R. Sutton, F. Ammirati et al., "Guidelines for the diagnosis and management of syncope (version 2009)," European Heart Journal, vol. 30, no. 21, pp. 2631-2671, 2009.

[8] M. Zamani, M. Esmailian, and Z. Yoosefian, "QT interval in pregnant and non-pregnant women," Emergency, vol. 2, no. 1, pp. 22-25, 2014.

[9] M. Linzer, E. H. Yang, N. A. M. Estes III, P. Wang, V. R. Vorperian, and W. N. Kapoor, "Clinical guideline: diagnosing syncope: part 1: value of history, physical examination, and electrocardiography," Annals of Internal Medicine, vol. 126, no. 12, pp. 989-996, 1997.

[10] P. Alboni, M. Brignole, C. Menozzi et al., "Diagnostic value of history in patients with syncope with or without heart disease," Journal of the American College of Cardiology, vol. 37, no. 7, pp. 1921-1928, 2001.

[11] G. Costantino, F. Perego, F. Dipaola et al., "Short- and longterm prognosis of syncope, risk factors, and role of hospital admission: results from the STePS (Short-Term Prognosis of Syncope) study," Journal of the American College of Cardiology, vol. 51, no. 3, pp. 276-283, 2008.

[12] A. Del Rosso, A. Ungar, R. Maggi et al., "Clinical predictors of cardiac syncope at initial evaluation in patients referred urgently to a general hospital: the EGSYS score," Heart, vol. 94, no. 12, pp. 1620-1626, 2008.

[13] J. Quinn, D. McDermott, I. Stiell, M. Kohn, and G. Wells, "Prospective validation of the San Francisco Syncope Rule to predict patients with serious outcomes," Annals of Emergency Medicine, vol. 47, no. 5, pp. 448-454, 2006.

[14] J. V. Quinn, I. G. Stiell, D. A. McDermott, K. L. Sellers, M. A. Kohn, and G. A. Wells, "Derivation of the San Francisco syncope rule to predict patients with short-term serious outcomes," Annals of Emergency Medicine, vol. 43, no. 2, pp. 224-232, 2004.

[15] M. J. Reed, D. E. Newby, A. J. Coull, K. G. Jacques, R. J. Prescott, and A. J. Gray, "The risk stratification of syncope in the emergency department (ROSE) pilot study: a comparison of existing syncope guidelines," Emergency Medicine Journal, vol. 24, no. 4, pp. 270-275, 2007.

[16] M. J. Reed, D. E. Newby, A. J. Coull, R. J. Prescott, K. G. Jacques, and A. J. Gray, "The ROSE (risk stratification of syncope in the emergency department) study," Journal of the American College of Cardiology, vol. 55, no. 8, pp. 713-721, 2010.

[17] V. Thiruganasambandamoorthy, E. P. Hess, A. Alreesi, J. J. Perry, G. A. Wells, and I. G. Stiell, "External validation of the San Francisco syncope rule in the Canadian setting," Annals of Emergency Medicine, vol. 55, no. 5, pp. 464-472, 2010.

[18] J. Plaseka, V. Doupalb, J. Fürstovac, and A. Martineka, "The EGSYS and OESIL risk scores for classification of cardiac etiology of syncope: comparison, revaluation, and clinical implications," Biomedical Papers, vol. 154, no. 2, pp. 169-173, 2010.

[19] A. Ungar, A. Del Rosso, F. Giada et al., "Early and late outcome of treated patients referred for syncope to emergency department: the EGSYS 2 follow-up study," European Heart Journal, vol. 31, no. 16, pp. 2021-2026, 2010.

[20] K. Kayayurt, H. Akoglu, O. Limon et al., "Comparison of existing syncope rules and newly proposed anatolian syncope rule to predict short-term serious outcomes after syncope in the Turkish population," International Journal of Emergency Medicine, vol. 5, article 17, 2012.

[21] S. Saedi, S. Oraii, and F. Hajsheikholeslami, "A cross sectional study on prevalence and etiology of syncope in Tehran," Acta Medica Iranica, vol. 51, no. 10, pp. 715-719, 2013. 


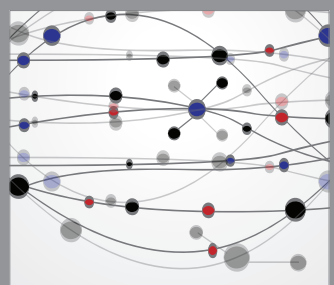

The Scientific World Journal
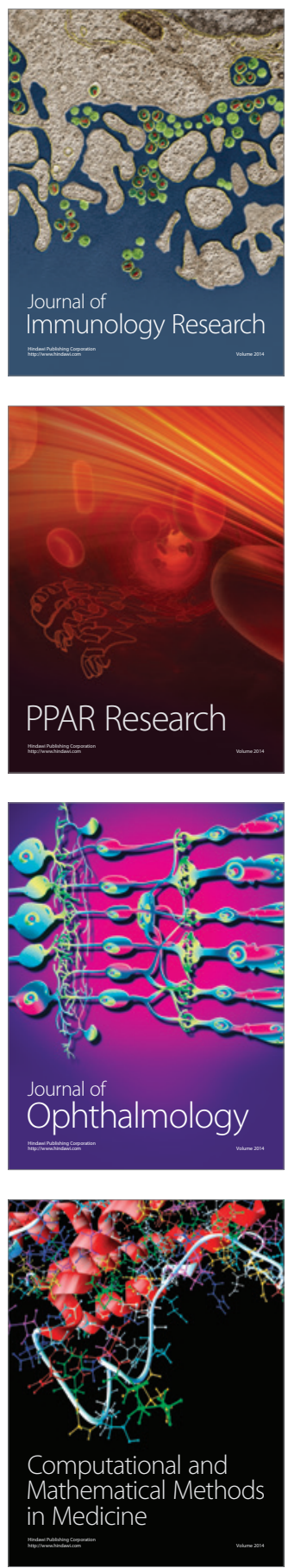

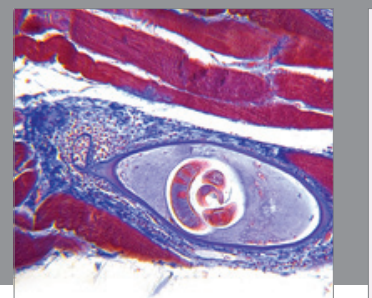

Gastroenterology

Research and Practice
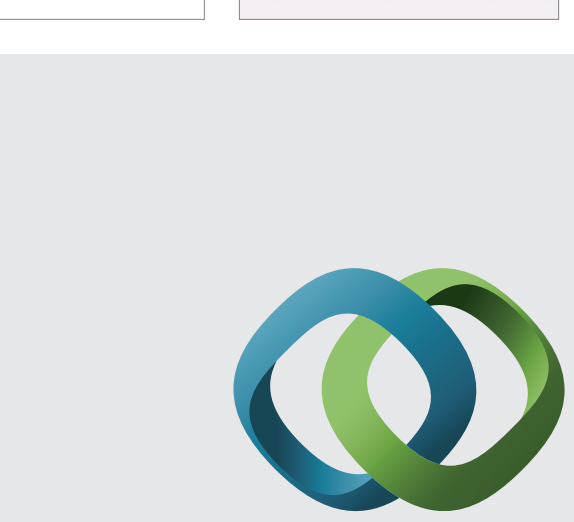

\section{Hindawi}

Submit your manuscripts at

http://www.hindawi.com
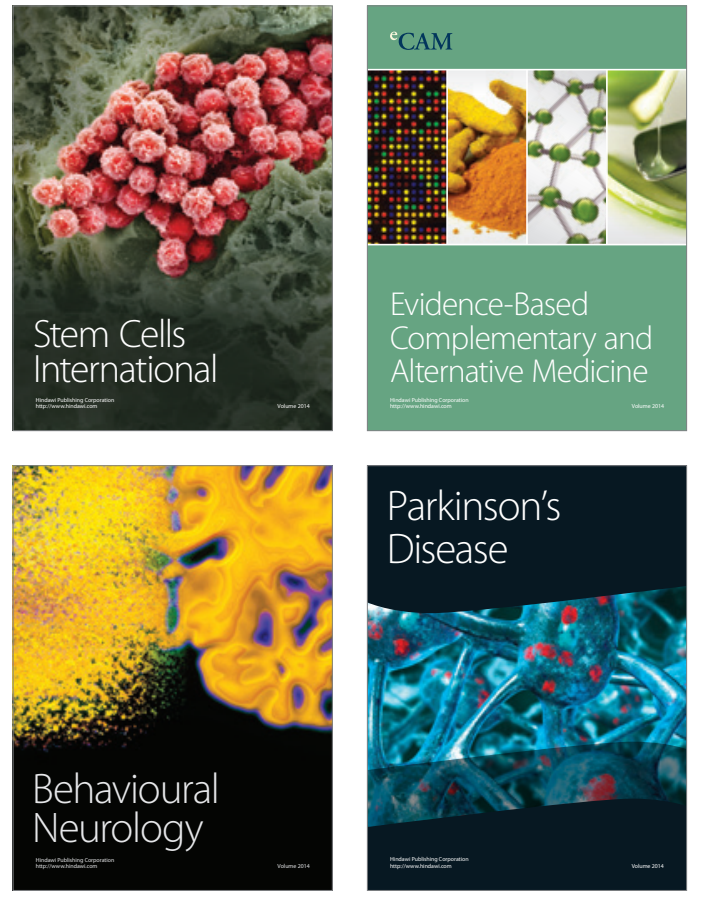
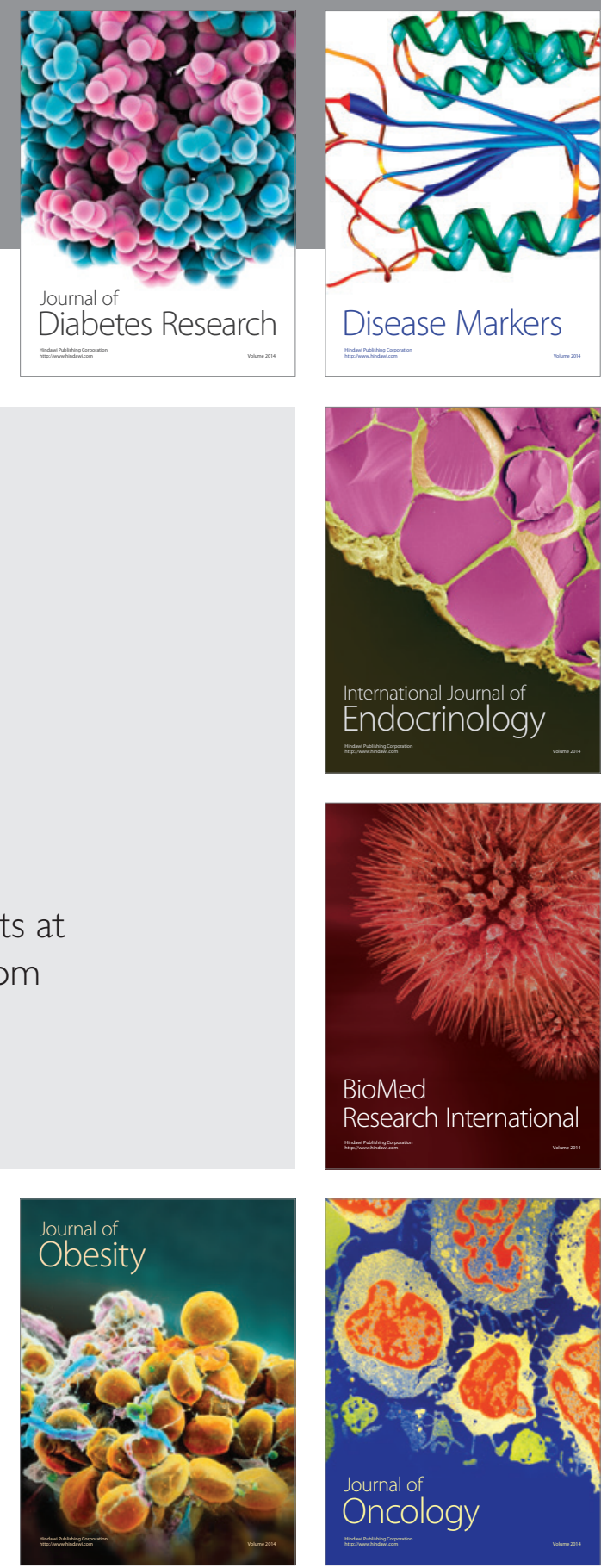

Disease Markers
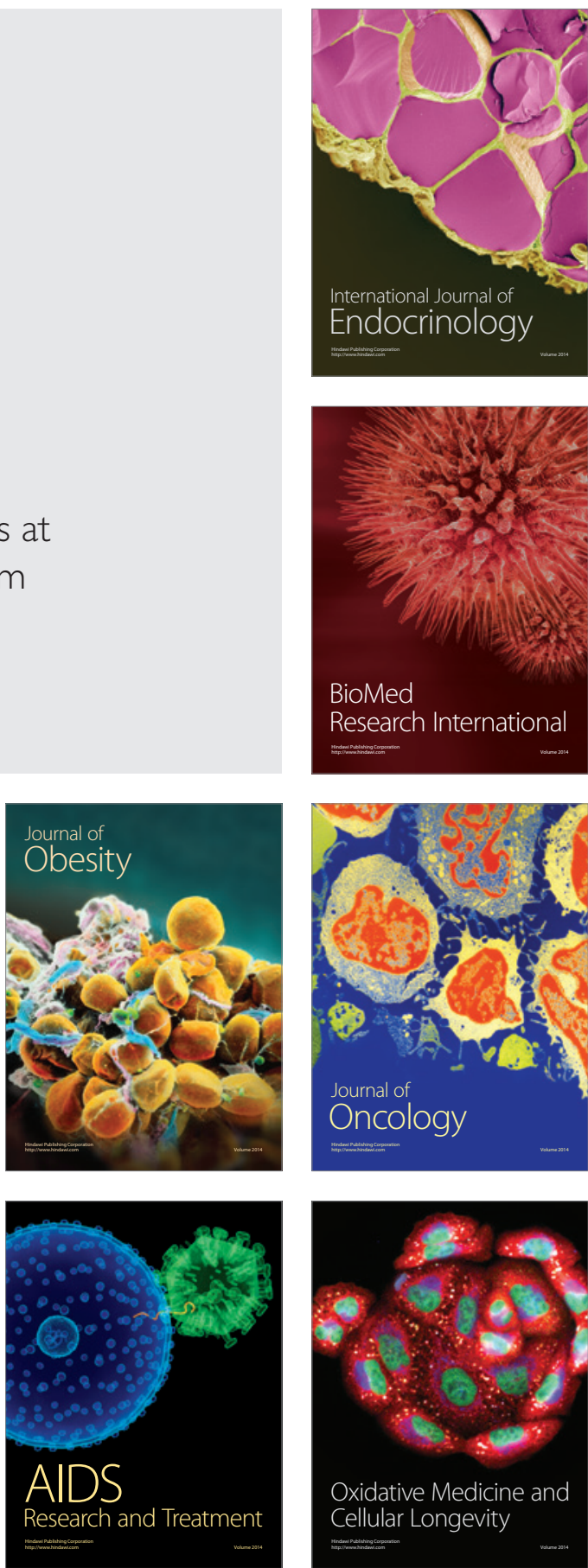